\title{
Ein bisschen besserer Sex dank transdermalem Östrogen
}

\author{
Östrogene, transdermal verabreicht, können dazu beitragen, dass Frauen in der frühen Postmenopause \\ wieder mehr Freude am Sex haben. Der Nutzen nimmt sich allerdings vergleichsweise bescheiden aus.
}

_ Das Female Sexual Function Inventory (FSFI) fragt Frauen nach Verlangen, Erregung, vaginaler Befeuchtung, Orgasmusfähigkeit, Zufriedenheit und Schmerzen beim Verkehr. Es gibt 0 bis 36 Punkte - je mehr, desto besser.

Mit dem FSFI bewehrt haben Ärzte von der Yale School of Medicine in New Haven, Connecticut, untersucht, ob sich bei Frauen in der frühen Postmenopause die Sexualfunktion durch den Einsatz von Hormonen verbessern lässt. 670 Frauen (Durchschnittsalter 53 Jahre) nahmen daran teil. 204 von ihnen erhielten im randomisierten und doppelt verblindeten Verfahren transdermales 17-beta-Estradiol (50 $\mu \mathrm{g} / \mathrm{d}), 209$ konjugiertes equines Östrogen oral $(0,45$ mg/d) und 257 Placebo, im Fall der Hormongruppen monatlich zwölf Tage lang auch mikronisiertes Progesteron (200 mg). Die Studie lief über 48 Monate.
Signifikante Effekte gegenüber Placebo waren nur für transdermales Estradiol festzustellen, und auch nur bei Frauen mit zu Beginn geringer Sexualfunktion (FSFI-Score $\leq 26$ Punkte). Diese stellten allerdings mit mehr als $70 \%$ in allen Gruppen die Mehrheit. Der mittlere Zugewinn beim FSFI-Wert betrug unter transdermalem Estradiol 3,9 Punkte.

- rb

- Taylor HS et al. JAMA Internal Med 2017, online 28. August

\section{Wie oft, mit wem und wie?}

\section{Sex-Studie klärt Deutschland auf}

\section{Wie viele Deutsche verwenden ein Kondom? Haben Männer oder Frauen mehr Sexpartner? Eine Studie liefert die Fakten.}

Deutsche

Männer haben nach eigenen Angaben im Schnitt schon mit zehn Partnerinnen geschlafen - Frauen dagegen nur mit fünf Partnern. So lautet ein Ergebnis einer repräsentativen Studie zum Sexualverhalten in Deutschland. 2.524 Menschen ab 14 Jahren waren per Fragebogen um Auskunft gebeten worden.

Auch bei der Treue zeigten sich Unterschiede zwischen den Geschlechtern: Jeder fünfte Mann gab an, in einer Partnerschaft schon einmal fremdgegangen zu sein. Bei den Frauen betrug der Anteil $15 \%$.
Weitere Erkenntnisse: $88 \%$ der Männer und $89 \%$ der Frauen hatten in ihrem Leben Vaginalverkehr. Oral befriedigt wurden schon einmal 56\% der Männer und $48 \%$ der Frauen. Mehr als die Hälfte der Befragten (57\%) hatte einen festen Partner. $86 \%$ der Männer und $82 \%$ der Frauen gaben an, heterosexuell zu sein. 5\% der Männer und 8\% der Frauen hatten schon einmal gleichgeschlechtliche Kontakte. Jeweils $1 \%$ gab an, rein homosexuell zu sein.

$76 \%$ der Paare verhüten in ihrer Beziehung nie mit Kondomen. Jede zweite Frau, die 50 Jahre oder jünger ist, nimmt die Pille oder ähnliche orale Verhü- tungsmittel. 5\% verzichten wegen Kinderwunsch auf Verhütung.

Sorgen machte den Forschern eine kleine Gruppe der Befragten (2,5\%): Sie gaben an, während ihrer aktuellen Beziehung sexuelle Außenkontakte gehabt zu haben und das auch schon ohne Kondom. Diese Personen hatten außerdem dreimal so viele Sexpartner wie der Durchschnitt: Männer 38, Frauen 17. Die Studienautoren raten dringend: Wer bei wechselnden Sexpartnern nicht ständig Kondome benutzt, sollte sich regelmäßig sexualmedizinisch untersuchen lassen.

- J Haversath et al. Dtsch Arztebl Int 2017;114(33-34):545-50 